\title{
Regulation of Antioxidant Stress-Responsive Transcription Factor Nrf2 Target Gene in the Reduction of Radiation Damage by the Thrombocytopenia Drug Romiplostim
}

\author{
Akane Chiba, ${ }^{a}$ Nanami Kawabata,${ }^{a}$ Masaru Yamaguchi, ${ }^{a}$ Shinji Tokonami, ${ }^{b}$ and \\ Ikuo Kashiwakura*,a \\ ${ }^{a}$ Department of Radiation Science, Hirosaki University Graduate School of Health Sciences; 66-1 Hon-cho, \\ Hirosaki, Aomori 036-8564, Japan: and ${ }^{b}$ Institute of Radiation Emergency Medicine Hirosaki University; 66-1 Hon- \\ cho, Hirosaki, Aomori 036-8564, Japan.
}

Received May 23, 2020; accepted September 3, 2020

Ionizing radiation induces severe oxidative stress, resulting in individual death by acute radiation syndrome. The nuclear factor-erythroid-2-related factor 2 (Nrf2) plays an important role in the antioxidant response pathway. Recently, romiplostim (RP), an idiopathic thrombocytopenic purpura therapeutic drug, was reported to completely rescue mice exposed to lethal total-body irradiation (TBI). However, the details underlying the mechanism for reducing radiation damage remain largely unknown. To elucidate the involvement of the master redox regulator Nrf2 in the radio-mitigative efficacy of $R P$ on TBI-induced oxidative stress, expression of Nrf2 target genes in hematopoietic tissues such as bone marrow, spleen, and lung from mice treated with RP for three consecutive days after 7 Gy of X-ray TBI was analyzed. RP promoted the recovery of bone marrow cells from day 10 and the significant up-regulation of reduced nicotinamide adenine dinucleotide phosphate (NAD(P)H) dehydrogenase quinone 1 (Nqo1), glutamate-cysteine ligase catalytic subunit $(G c l c)$ and glutamate-cysteine ligase modifier subunit $(\mathrm{Gclm})$ was observed compared to the TBI mice. RP also promoted the recovery of splenic cells on day 18, and the significant up-regulation of Nqo1, Gclc and $G c l m$ in spleen both on day 10 and 18 and $N q o 1$ and Gclm in lung on day 10 was observed compared to the TBI mice. The present study suggests that the radio-mitigative effects of RP indicates on the activation of Nrf2 target genes involved in redox regulation and the antioxidative function, especially Nqo1, Gclc and Gclm. It is indicating the importance of these genes in the maintenance of biological homeostasis in response to radiation-induced oxidative stress.

Key words accute radiation syndrome; romiplostim; radio-mitigative effect; nuclear factor-erythroid2-related factor 2 (Nrf2) target gene; reduced nicotinamide adenine dinucleotide phosphate dehydrogenase quinone 1 (Nqol); glutamate-cysteine ligase modifier subunit ( Gclm)

\section{INTRODUCTION}

Ionizing radiation is an external oxidative stress factor, that can cause cell death and organ damage by exceeding the capacity of the antioxidant response system, resulting in individual death by acute radiation syndrome (ARS), depending on the dose. Low-linear-energy-transfer ionizing radiation, such as X-rays and $\gamma$-rays, generate reactive oxygen species (ROS) that can lead to biological damage and alter cellular signalling pathways, which are involved in cell cycle arrest, DNA damage, cell death apoptosis, and potentially carcinogenesis. ${ }^{1,2)}$

Cells are equipped with various antioxidant systems to biologically defend against ROS. Several previous studies have shown that the nuclear factor-erythroid-2-related factor 2 (Nrf2) plays an important role in the antioxidant response pathway against oxidative stress. ${ }^{3-7)}$ Under physiological conditions, Nrf2 is sequestered in the cytoplasm by kelch-like ECH-associated protein 1 (Keap1)-mediated ubiquitination and proteasome-dependent degradation. ${ }^{8)}$ However, under oxidative stress, detachment of Nrf2 from Keap1 is affected by ROS and translocate to the nucleus, following which, binds to antioxidant response elements in the promoter regions of its target genes involved in redox regulation and antioxidative functions. ${ }^{3)}$ We recently reported the relationship between radiation doses ranging from 0.5 to $3 \mathrm{~Gy}$ and the expression of $7 \mathrm{Nrf} 2$ target genes involved in oxidative stress, namely heme oxygenase 1 ( $\mathrm{Ho}-1)$, ferritin heavy polypeptide 1 (Fthl), reduced nicotinamide adenine dinucleotide phosphate (NAD(P)H) dehydrogenase quinone 1 (Nqol), glutamatecysteine ligase catalytic subunit $(\mathrm{Gclc})$, glutamate-cysteine ligase modifier subunit $(G c l m)$, glutathione reductase $(G s r)$, and thioredoxin reductase 1 (Txnrd1), ${ }^{9)}$ suggesting a statistically significant positive correlation between the radiation dose and the mRNA expression of Fthl, which is the major intracellular iron storage protein in prokaryotes and eukaryotes. These findings suggested the potential utility of these target genes as bio-dosimeters and/or damage markers in individuals exposed to ionizing radiation.

Our recent study showed that the thrombopoietin receptor (TPOR) agonist romiplostim (RP), an idiopathic thrombocytopenic purpura therapeutic drug, completely rescued mice exposed to lethal $\gamma$-total-body irradiation (TBI) suggesting that RP may not only promote hematopoiesis in various organs in irradiated individuals but also mitigate the dysfunction or regenerate the original function in multiple organs. ${ }^{10}$ The radio-mitigative effects of RP have also been reported by other groups. $^{11,12)}$ Especially, Wong et al. have demonstrated the radiation damage reduction effect of $\mathrm{RP}$ in non-human 
primates. $^{12)}$ However, the mechanism of action of RP cannot be explained only by signal transduction via TPOR, and the involvement of antioxidant mechanism is also speculated.

In the present study, we evaluated the expression of $\mathrm{Nrf} 2$ target genes in the bone marrow, spleen and lung, which are important in hematopoietic function, using a mouse model of lethal dose TBI (7Gy). The lungs have been reported to be a major site of terminal platelet production in mice and an organ with considerable hematopoietic potential. ${ }^{13)}$ In particular, we evaluated the involvement of RP administration in Nrf2 target gene expression on days 10 and 18 after TBI, which is an important branching point in damage repair and recovery from individual death.

\section{MATERIALS AND METHODS}

Experimental Animals and X-Ray TBI Female C57BL/ $6 \mathrm{~J}$ Jcl mice at 7 weeks of age were obtained from Clea Japan (Tokyo, Japan). At 8 weeks of age, mice were subjected to TBI with $7 \mathrm{~Gy}(150 \mathrm{kVp}, 20 \mathrm{~mA}, 0.5-\mathrm{mm}$ aluminium and $0.3-\mathrm{mm}$ copper filters) at a dose rate of $1.0 \mathrm{~Gy} / \mathrm{min}$ using an MBR-1520R X-ray generator (Hitachi Medical, Tokyo, Japan). This equipment used an ionization chamber dosimeter as the measuring instrument and was set so that the cumulative dose was $7 \mathrm{~Gy}$. All of the mice were housed in standard cages in a temperature-controlled room under a 12-h light/dark cycle. Mice were provided with sterilized standard laboratory mouse chow diet and drinking water ad libitum. All experiments were conducted according to legal regulations in Japan and the Guidelines for Animal Experiments of Hirosaki University after approval by the animal experimental committee. The selection criteria applied prior to sacrifice were more than $20 \%$ loss of body weight, respiratory distress. The number of mice used for each experiment is indicated in the figure legends.

Mitigator Treatment Within $2 \mathrm{~h}$ after TBI, human TPOR agonist RP (Romiplate ${ }^{\circledR}$; Kyowa Hakko Kirin, Tokyo, Japan) was intraperitoneally administered at almost the same time for 3 consecutive days, and the daily dosage of RP was $50 \mu \mathrm{g} / \mathrm{kg}$ of body weight based on our previous reports. ${ }^{10,12,13)}$ In addition to the above mice that received TBI and RP administration (TBI + RP mice), mice treated with TBI only (TBI mice), those treated with RP only (RP mice), and those that did not receive either TBI or RP (control mice) were also evaluated in this study. TBI and control mice received the normal saline solution (Otsuka Pharmaceutical, Tokyo, Japan) used to prepare the drugs. At days 10 and 18 after TBI, each mouse was anaesthetized with isoflurane (Powerful Isoful ${ }^{\circledR}$; Zoetis, London, U.K.) and sacrificed for collection of the bone marrow, spleen and lung.

Collection of Bone Marrow Cells, Splenic Cells and Lung Bone marrow cells (BMCs) were harvested from both femurs by flushing with ethylenediaminetetraacetic acidphosphate buffered-saline, $\mathrm{pH} 7.4$, containing $0.5 \%$ bovine serum albumin. Spleens were placed in Hanks' balanced salt solution and mechanically dissociated. Minced lungs were placed in $2 \mathrm{~mL}$ phosphate buffered saline with $5 \mu \mathrm{L} / \mathrm{mL}$ deoxyribonuclease (DNase) I (Roche, Basel, Switzerland) and $0.5 \mathrm{mg} / \mathrm{mL}$ Liberase (Roche) and then incubated at $37^{\circ} \mathrm{C}$ for $30 \mathrm{~min}$. The above cell suspensions were filtered through a $45-\mu \mathrm{m}$ strainer and haemolyzed in Gey's salt solution for red blood cell lysis. Viable cells were counted using a haemocy- tometer (BurkerTurk; Sunlead Glass, Saitama, Japan) with the trypan blue dye exclusion method (Sigma-Aldrich ${ }^{\circledR}$; St. Louis, MO, U.S.A.).

Total RNA Extraction The following seven Nrf2 target genes were analysed in the present study: Ho-1, Fth1, Nqol, Gclc, Gclm, Gsr and Txnrd1. RNA, including microRNA (miRNA), was extracted from BMCs, splenic, and lung cells using an miRNeasy Mini Kit (QIAGEN, Hilden, Germany) according to the manufacturer's instructions. The quality and concentration of the yielded RNA were assessed using the NanoDrop spectrophotometer (NanoDrop Technologies, Wilmington, DE, U.S.A.). All RNA samples had 260/280 nm absorbance ratios of 1.8-2.0.

Quantitative (q)RT-PCR First-standard cDNA from RNA was synthesized using the SuperScript ${ }^{\mathrm{TM}}$ IV VILO ${ }^{\mathrm{TM}}$ Master Mix with ezDNase (Thermo Fisher Scientific ${ }^{\mathrm{TM}}$ Inc., Boston, MA, U.S.A.) according to the manufacturer's instructions. qRT-PCR was performed using the Power SYBER ${ }^{\circledR}$ Green Master Mix (Applied Biosystems, Carlsbad, CA, U.S.A.) and a StepOnePlus ${ }^{\mathrm{TM}}$ Real-Time PCR System (Thermo Fisher Scientific). ATPase subunit 6 mRNA (ATP6) was used as an internal control for all reactions because the fluctuation of ATP6 was the lowest among 16 housekeeping genes and 16 mouse orthologs of human internal standard genes analysed with the TaqMan ${ }^{\circledR}$ Array Mouse Endogenous Control 96-well Plate (Thermo Fisher Scientific) in the preliminary test. We performed qPCR with the following typical amplification parameters: $95^{\circ} \mathrm{C}$ for $10 \mathrm{~min}$, followed by 40 cycles at $95^{\circ} \mathrm{C}$ for 15 -s and $60^{\circ} \mathrm{C}$ for $1 \mathrm{~min}$. Relative differences in the gene expression were determined by the $\Delta \Delta \mathrm{CT}$ method. The mRNA expression of control mice was defined as the baseline. The oligonucleotide primer sets used in this analysis and the internal control ATP6 were purchased from Eurofins Genomics Inc. (Tokyo, Japan) (Table 1).

Statistical Analyses Data are represented as the mean \pm standard deviation (S.D.). The levels of significance were calculated using the Excel 2016 software program (Microsoft, Redmond, WA, U.S.A.) with the Statcel 3 add-on (OMS, Saitama, Japan) and Origin 7.5 (OriginLab, Northamp-

Table 1. Sequences of PCR Primers

\begin{tabular}{|c|c|}
\hline Gene & Primer sequence $\left(5^{\prime}\right.$ to $\left.3^{\prime}\right)$ \\
\hline \multirow[t]{2}{*}{ Ho-1 } & F: AGGGTCAGGTGTCCAGAGAA \\
\hline & R: CTTCCAGGGCCGTGTAGATA \\
\hline \multirow[t]{2}{*}{ Fthl } & F: TGGAGTTGTATGCCTCCTACG \\
\hline & R: TGGAGAAAGTATTTGGCAAAGTT \\
\hline \multirow[t]{2}{*}{ Nqol } & F: AGCGTTCGGTATTACGATCC \\
\hline & R: AGTACAATCAGGGCTCTTCTCG \\
\hline \multirow[t]{2}{*}{ Gclc } & F: AGATGATAGAACACGGGAGGAG \\
\hline & R: TGATCCTAAAGCGATTGTTCTTC \\
\hline \multirow[t]{2}{*}{ Gclm } & F: TGACTCACAATGACCCGAAA \\
\hline & R: TCAATGTCAGGGATGCTTTCT \\
\hline \multirow[t]{2}{*}{ Gsr } & F: ACTATGACAACATCCCTACTGTGG \\
\hline & R: CCCATACTTATGAACAGCTTCGT \\
\hline \multirow[t]{2}{*}{ Txnrdl } & F: TCTGAAGAAAAAGCCGTAGAGAA \\
\hline & R: TTCCAATGGCCAAAAGAAAC \\
\hline \multirow[t]{2}{*}{ ATP6 } & F: CCATAAATCTAAGTATAGCCATTCCAC \\
\hline & R: AGCTTTTTAGTTTGTGTCGGAAG \\
\hline
\end{tabular}

F; forward primer, R; reverse primer. 
ton, MA, U.S.A.). $p$ Values less than 0.05 were considered to indicate statistical significance. The data were analysed with one-way ANOVA and Tukey-Kramer or Bonferroni/Dunn multiple comparison tests.

\section{RESULTS}

Changes of Body Weight in the Surviving Mice after Lethal TBI We previously showed that the TPOR agonist RP completely rescued mice exposed to lethal TBI. ${ }^{10,12,13)}$ To clarify the relationship between survival time of mice and changes of the body weight after lethal TBI, both values were plotted on Fig. 1. TBI mice treated without RP lost body weight gradually and died within day 25 , but all TBI plus RP mice survived on day 30 and gained 10 to $20 \%$ of their body weight compared to those at the time of TBI. No other symptoms of ARS such as hair loss or dermatitis were observed (data not shown).

Changes in the Number of Viable Cells in Bone Marrow, Spleen and Lung The numbers of viable cells in each organ on days 10 and 18 are shown in Fig. 2. BMCs and splenic cells were markedly reduced by TBI on both days. RP administration significantly increased BMCs but did not recover to the same levels as in control mice. For splenic cells, the effects of RP were not observed on the 10th day, but on the 18th day,

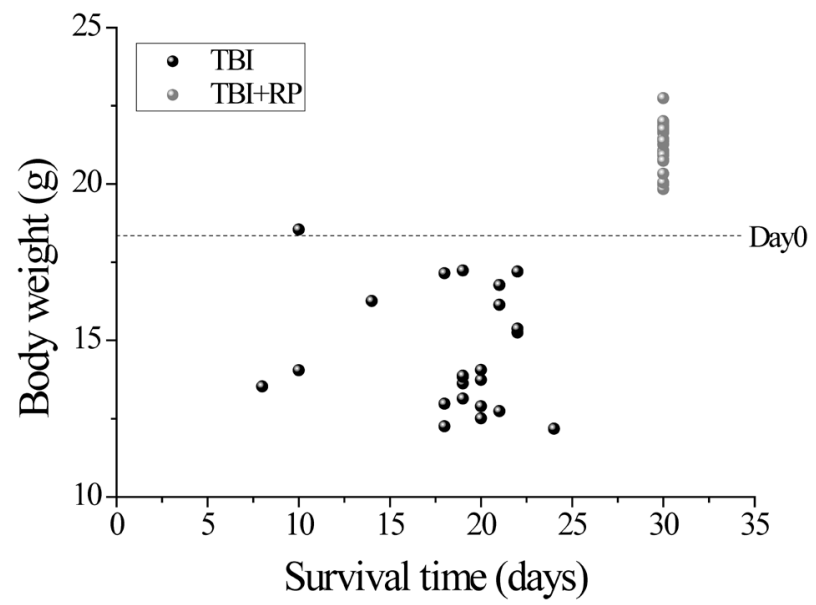

Fig. 1. Relationship between Survival Time of Mice and Changes of the Body Weight

Mice were intraperitoneally administered RP $(50 \mu \mathrm{g} / \mathrm{kg}$ of body weight $/ \mathrm{d})$ for 3 conclusive days within $2 \mathrm{~h}$ after exposure to a 7 Gy dose of TBI $(n=20$, gray circle). Mice treated with TBI only received injections of the normal saline solution as vehicle $(n=23$, black circle). The $X$-axis and $Y$-axis in this plot represent the survival time after TBI and body weight, respectively, and the dotted line shows the initial average value of body weight of control mice on day 0 . the number of cells recovered to the level in control mice. In contrast, the numbers of lung cells were not affected by TBI or RP at all.

The Expression of Nrf2 Target Genes Detected in BMCs, Splenic and Lung Cells The mRNA expression of Ho-1, Fth1, Nqol, Gclc, Gclm, Gsr and Txnrd1 detected in BMCs, splenic and lung cells was analysed at 10th and 18th days after TBI. The results are summarized in Table 2. TBI decreased the expression of Gclc and Gsr in BMCs, increased Ho-1, Fth1, Gsr and Txnrdl in the splenic cells, and decreased expression of Ho-1, Fthl and Gsr in the lung cells. All of these expressions were regulated to the initial level by

Day 10

Day18
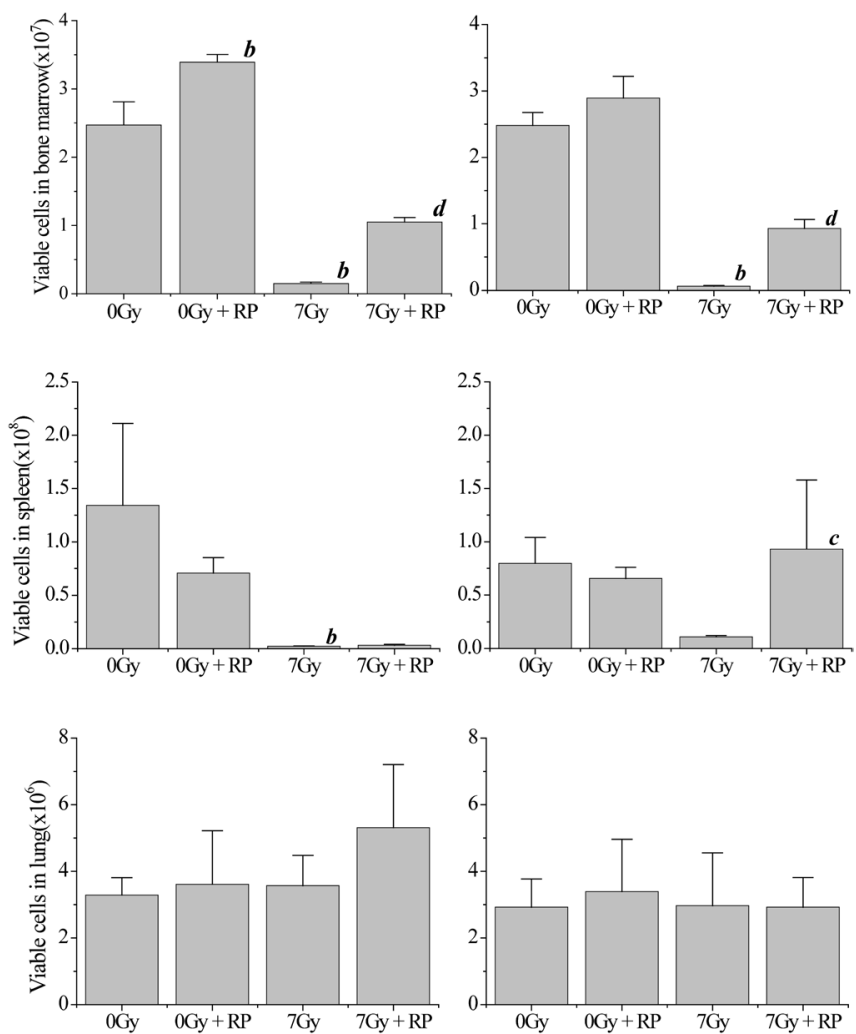

Fig. 2. RP Mitigates TBI-Induced Hematopoietic Damage in Mice

Mice were sham-irradiated as controls or irradiated with 7Gy TBI and then treated with vehicle or RP as described in the text. Total viable cell numbers in the bone marrow, spleen and lung observed in the TBI and control mice with or without RP treatment until day 10 and 18 post-TBI ( $n=4$ per group). $a ; p<0.05$ vs. $0 \mathrm{~Gy}, b ; p<0.01$ vs. $0 \mathrm{~Gy}, c ; p<0.05$ vs. $7 \mathrm{~Gy}, d ; p<0.01$ vs. $7 \mathrm{~Gy}$. The data are analyzed using the Bonferroni/Dunn test.

Table 2. Summary of the Changes in the Nrf2 Target Gene Expression Following Lethal TBI and RP Administration

\begin{tabular}{|c|c|c|c|c|c|c|c|c|}
\hline & Days & Ho-1 & Fthl & Nqo1 & Gclc & Gclm & $G s r$ & Txnrd1 \\
\hline \multirow[t]{2}{*}{ Bone marrow } & 10 &,$- \downarrow *$ &,-- &,-- &,-- &,-- & $\downarrow * *,-$ &,-- \\
\hline & 18 &,-- & - - $*$ & $-\uparrow * *$ & $\downarrow * *, \uparrow * *$ & $-\uparrow * *$ & $\downarrow * *,-$ &,-- \\
\hline \multirow[t]{2}{*}{ Spleen } & 10 & $\uparrow * *,-$ & $\uparrow * *,-$ & $-\uparrow * *$ & 一, $\uparrow * *$ &,$- \uparrow *$ & 一, $\uparrow * *$ & $\uparrow *,-$ \\
\hline & 18 & $\uparrow * *, \downarrow * *$ &,$- \uparrow * *$ & $-\uparrow * *$ &,$- \uparrow * *$ &,$- \uparrow * *$ & $\uparrow *$ &,-- \\
\hline \multirow[t]{2}{*}{ Lung } & 10 & $\downarrow * *, \uparrow *$ & $\downarrow *,-$ & $-\uparrow * *$ &,-- & $-\uparrow * *$ & $\downarrow *,-$ &,-- \\
\hline & 18 &,-- &,-- &,-- &,-- &,-- & $\downarrow * *,-$ &,-- \\
\hline
\end{tabular}

The symbols in each box indicate the fluctuations of the gene expression depending on the degree of statistical significance of the difference between mice with or without TBI, or TBI mice with or without RP treatment, respectively $(* p<0.05$ and $* * p<0.01)$. Up- or down-arrow mean the up- or down-regulated gene expression, respectively. 

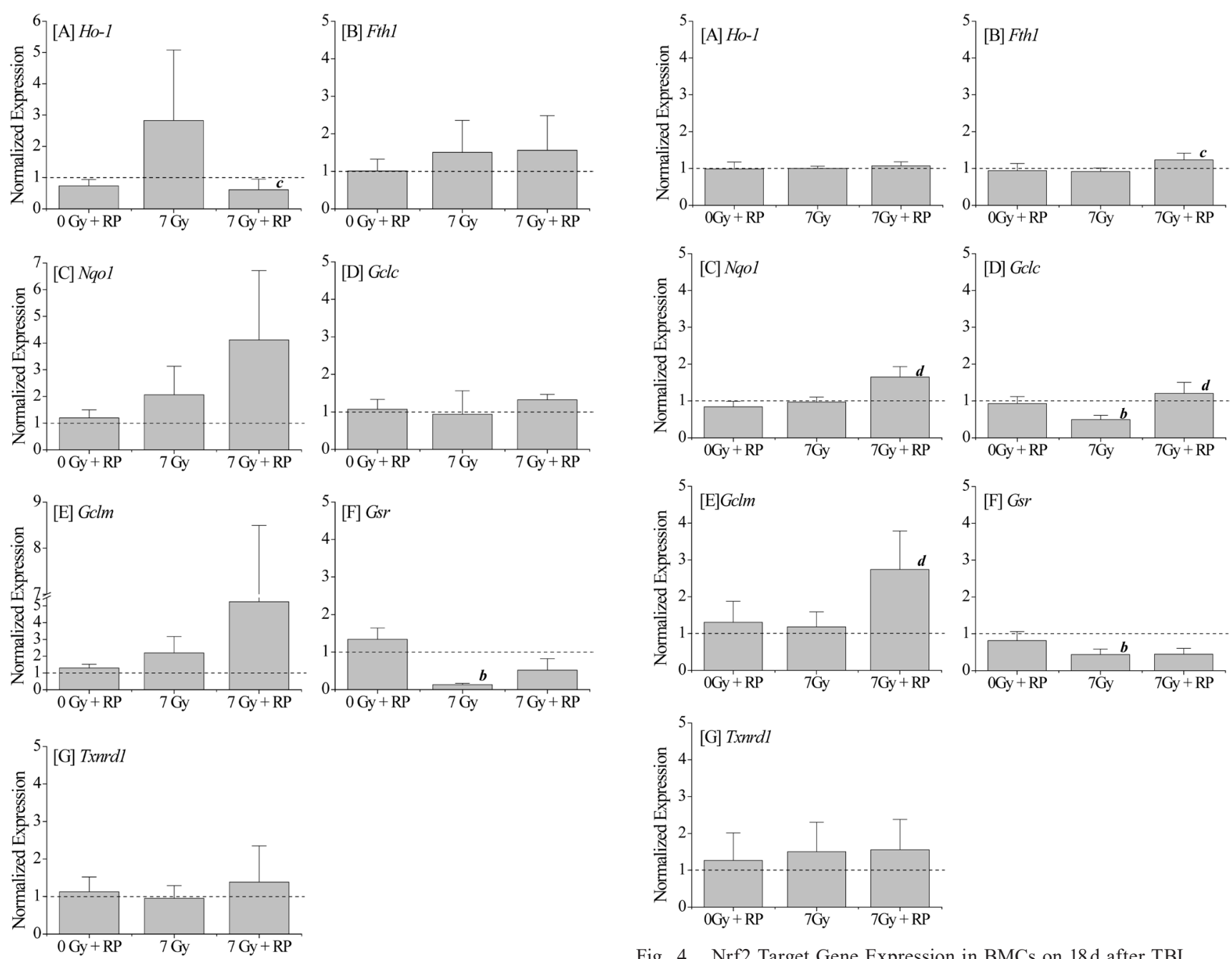

Fig. 3. Nrf2 Target Gene Expression in BMCs on $10 \mathrm{~d}$ after TBI

The dotted line in the figure shows the value for control mice. Total RNA from bone marrow was examined by qRT-PCR for the following genes: Ho-l, Nqol, Gclc, Gclm, Gsr, and Txnrdl. The mRNA level in control mice was defined as the baseline. ATP6 was used as an internal control. $a ; p<0.05 v s$. $0 \mathrm{~Gy}, b ; p<0.01 v s$. $0 \mathrm{~Gy}, c ; p<0.05$ vs. $7 \mathrm{~Gy}, d ; p<0.01$ vs. $7 \mathrm{~Gy}$ were considered to indicate statistical significance, which were analyzed with one-way ANOVA and Bonferroni/Dunn multiple comparison tests.

RP administration. Furthermore, in BMCs, Nqol and Gclm increased more than the irradiation level by RP administration on both days (Figs. 3, 4).

In splenic cells, the expression of Nqol, Gclc, Gclm, and Gsr was significantly increased in TBI plus RP mice on day 10 (Fig. 5). On day 18, the expression of Fthl was also increased in addition to Nqol, Gclc, and Gclm (Fig. 6). However, the expression of Ho-1, Fth1 and Txnrdl, which were increased at $10 \mathrm{~d}$ after TBI, and Gsr at $18 \mathrm{~d}$ was not changed by RP treatment (Figs. 5, 6).

In lung cells on day 10 , TBI significantly decreased the expression of Ho-1, Fthl, and Gsr. These reductions in expression were improved by RP (Fig. 7). In particular, the expression of $\mathrm{NqO}$ and $\mathrm{Gclm}$ increased to a greater level than that see in the control cells. Similar upregulation was not observed on day 18 (Fig. 8).

\section{DISCUSSION}

In the present study, the response of transcription factor

Nrf2 target genes (Ho-1, Fth1, Nqol, Gclc, Gclm, Gsr, and Txnrd1), which are involved in redox regulation and antioxidative functions, was evaluated in mice exposed to a lethal dose of TBI, and the effects of the TPOR agonist RP on the expression of these genes was examined. Even in lethally irradiated individuals, fluctuations in expression of all Nrf2 target genes measured in this study were observed. Our recent study demonstrated that a statistically significant positive correlation between radiation dose ranged from 0.5 to 3 Gy TBI and Fth 1 mRNA expression was detected. ${ }^{9)}$ While Gsr expression was significantly associated with spleen weight loss, splenic cell number reduction and BMCs apoptosis, no significant correlation was observed between Gsr expression and radiation dose. The present results showed that lethal TBI increased the expression of most genes in the spleen (Table 2, Figs. 5, 6). Oxidative stress dissociates the Nrf2-keap1 complex, leading to the translocation of Nrf2 to the nucleus. Nrf2 binds with the anti-oxidant responsive element present in the promoter region of anti-oxidants, detoxicants, and cytoprotective genes and regulates its target gene expression. ${ }^{3,14)}$ Therefore, although 

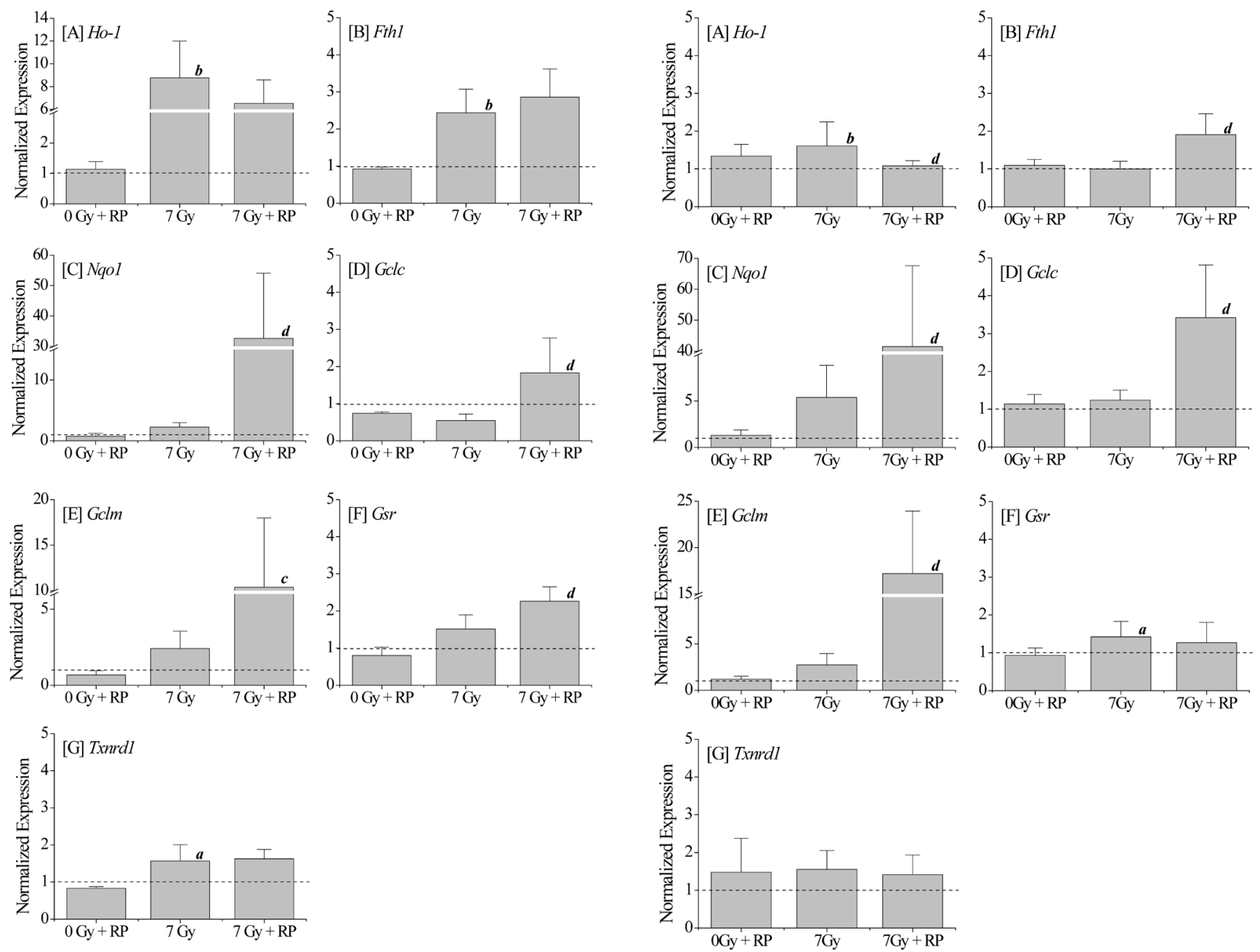

Fig. 5. Nrf2 Target Gene Expression in Splenic Cells on $10 \mathrm{~d}$ after TBI

The dotted line in the figure shows the value for control mice. Total RNA from spleen was examined by qRT-PCR for the following genes: Ho-1, Nqo1, Gclc, Gclm, Gsr, and Txnrdl. The mRNA level in control mice was defined as the baseline. ATP6 was used as an internal control. $a ; p<0.05$ vs. $0 \mathrm{~Gy}, b ; p<0.01$ vs. $0 \mathrm{~Gy}, c ; p<0.05$ vs. $7 \mathrm{~Gy}, d ; p<0.01$ vs. $7 \mathrm{~Gy}$ were considered to indicate statistical significance, which were analyzed with one-way ANOVA and Bonferroni/Dunn multiple comparison tests.

the Nrf2 target genes responded differently depending on the dose, these genes may play an important role in the maintenance of biological homeostasis in response to radiationinduced oxidative stress.

The administration of RP to mice exposed to lethal TBI resulted in an active recovery of the hematopoietic function and survival of mice. ${ }^{15,16)}$ In the present study, Nqol and Gclm were up-regulated in all tissues tested here (Figs. 3, 5, 7). Nqol catalyses the two-electron reduction of quinones and a wide range of other organic compounds. Its physiological role is believed to be partly the reduction of the free radical load in cells and the detoxification of xenobiotics. ${ }^{17,18)}$ It also has the non-enzymatic function of stabilizing a number of cellular regulators, including $\mathrm{p} 53 .{ }^{19,20)}$ Ross and Siegel suggested that Nqol binding to mRNA can regulate protein translation, and one of the important roles of Nqol when induced under oxidative stress conditions is maintaining the stability of major proteins. ${ }^{21)}$ The accumulation and activation of the tumour suppressor gene p53 induces growth arrest and apoptosis. In addition, Gclm, together with Gclc, constitutes GCL as a dimer of a modifier subunit having a regulating action and

Fig. 6. Nrf2 Target Gene Expression in Splenic Cells on $18 \mathrm{~d}$ after TBI

The dotted line in the figure shows the value for control mice. Total RNA from spleen was examined by qRT-PCR for the following genes: Ho-l, Nqol, Gclc, Gclm, Gsr, and Txnrd1. The mRNA level in control mice was defined as the baseline. ATP6 was used as an internal control. $a ; p<0.05$ vs. $0 \mathrm{~Gy}, b ; p<0.01$ vs. $0 \mathrm{~Gy}, c ; p<0.05 v s .7 \mathrm{~Gy}, d ; p<0.01 v s .7 \mathrm{~Gy}$ were considered to indicate statistical significance, which were analyzed with one-way ANOVA and Bonferroni/Dunn multiple comparison tests.

catalytic subunit having a catalytic action, respectively. ${ }^{22,23)}$ GCL is the rate-limiting enzyme responsible for the synthesis pathway of glutathione, which is the most important intracellular anti-oxidant. ${ }^{24,25)}$ When oxidative stress occurs in the living body, the transcriptional activity of the Gclc gene increases due to glutathione synthesis, which acts as a defense mechanism. ${ }^{26}$ ) The effect of RP on dysfunction was suggested to consequently affect the Nrf2 redox system and contribute to its target gene expression. Sekhar and Freeman introduced that Nrf2 is a critical factor for promoting survival of mammalian cells subjected to ionizing radiation due to its ability promotes the repair of DNA damage and drives detoxification of superoxide that is generated hours to days after irradiation. ${ }^{27)}$ In addition, crosstalk between the phosphoinositide 3-kinase (PI3K)-AKT and Nrf2 signalling pathways is capable of protecting cells against inflammatory and oxidative damage. $^{28)}$ Thabet and Mousetafa studied to evaluate the effect of rutin on PI3K/AKT-signalling in case of acrylamide or $\gamma$-irradiation-induced neurotoxicity. They found that rutin provides protection effect against acrylamide or $\gamma$-radiationinduced neurotoxicity via activation of the PI3K/AKT/ 

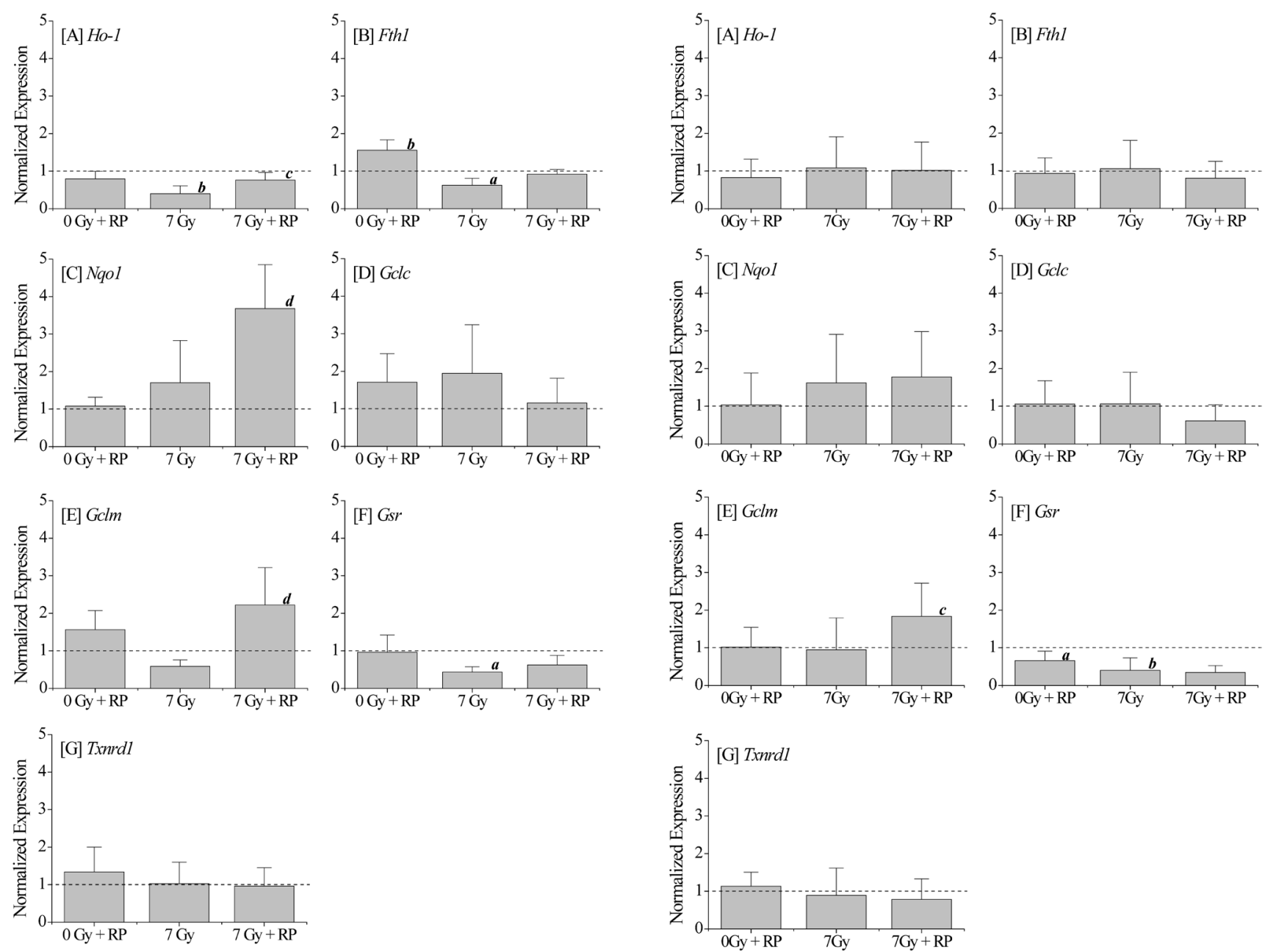

Fig. 7. Nrf2 Target Gene Expression in Lung Cells on $10 \mathrm{~d}$ after TBI

The dotted line in the figure shows the value for control mice. Total RNA from lung was examined by qRT-PCR for the following genes: Ho-1, Nqol, Gclc, Gclm, Gsr, and Txnrdl. The mRNA level in control mice was defined as the baseline. ATP6 was used as an internal control. $a ; p<0.05$ vs. $0 \mathrm{~Gy}, b ; p<0.01$ vs. $0 \mathrm{~Gy}, c$; $p<0.05$ vs. $7 \mathrm{~Gy}, d ; p<0.01$ vs. $7 \mathrm{~Gy}$ were considered to indicate statistical significance, which were analyzed with one-way ANOVA and the Turkey-Kramer $[\mathrm{A}, \mathrm{B}$, $\mathrm{F}]$ and Bonferroni/Dunn $[\mathrm{C}, \mathrm{E}]$ multiple comparison tests.

glycogen synthase kinase $3 \beta$ (GSK-3 $\beta$ )/Nrf2-pathway. ${ }^{29)}$ Rutin activated PI3K/AKT/GSK-3 $\beta /$ Nrf2-pathway through increased protein levels of $\mathrm{p}$-PI3K, $\mathrm{p}$-AKT and $\mathrm{p}-\mathrm{GSK}-3 \beta$ and upregulated the expression of $\mathrm{Nrf2}$. PI3K have an activating effect on Nrf2 translocation to the nucleus via the inhibition of GSK-3 $\beta$ by AKT phosphorylation which follows those therapies/diets/conditions that activate PI3K (upstream of AKT) ${ }^{30)}$ Binding of RP to TPOR causes the activation of Janus kinase 2 (JAK2) and signals from the phosphorylated molecules to the JAK2-signal transducer and activator of transcription 3 (STAT3)/STAT5 pathway, the mitogen-activated protein kinase (MAPK) pathway (extracellular signal-regulated kinase 1/2 (ERK1/2) pathway and p38 MAPK pathway), and the PI3K-AKT pathway. ${ }^{31)}$ Taken together, the present results suggest the possibility that RP involved in the activation of Nrf2, leading to the expression of Nqo1, Gclc and Gclm, particularly in the spleen, finally an increase in anti-oxidant activity.

There are many reports on compounds that activate Nrf2 target genes, including electrophilic substances. ${ }^{32-35)}$ Thus far, research on the development of radio-protective/radio-

Fig. 8. Nrf2 Target Gene Expression in Lung Cells on $18 \mathrm{~d}$ after TBI

The dotted line in the figure shows the value for control mice. Total RNA from lung was examined by qRT-PCR for the following genes: Ho-1, Nqol, Gclc, Gclm, Gsr, and Txnrdl. The mRNA level in control mice was defined as the baseline. ATP6 was used as an internal control. $a ; p<0.05$ vs. 0 Gy, $b ; p<0.01$ vs. 0 Gy, $c ; p<0.05 v s .7 \mathrm{~Gy}, d ; p<0.01 v s .7 \mathrm{~Gy}$ were considered to indicate statistical significance, which were analyzed with one-way ANOVA and the Turkey-Kramer multiple comparison tests.

mitigative agents has focused on various mechanisms of action, but few studies have targeted Nrf2. Furthermore, there are also few reports that RP promotes the expression of Nrf2 target genes. Recently, Lu et al. reported that 3,3'-diindolylmethane, a natural small molecule compound that is a potent antioxidant agent, increases the expression of $\mathrm{Nrf} 2$ and decreases the rate of apoptosis in the small intestine of irradiated mice. ${ }^{36)}$ Furthermore, Li et al. also reported that (-)-epicatechin exerts a protective effect on the intestine and promotes intestinal regeneration after radiation through Nrf2 and the Wnt/ $\beta$-catenin signal pathway. ${ }^{37)}$ Thus, the role of Nrf2 target genes in radiation injury reduction has been gradually elucidated. Preparing for the risk of high radiation exposure due to radiation/nuclear accidents or terrorism is an important issue in the era of nuclear energy era. Medical countermeasures for ARS, bone marrow death and intestinal death, are required. Therefore, more detailed studies clarifying the biological defence mechanism underlying the anti-oxidant response of transcription factor Nrf2 should be continued. 


\section{CONCLUSION}

The radio-mitigative effects of $\mathrm{RP}$ indicate on the activation of Nrf2 target genes involved in redox regulation and the antioxidative function, especially Nqol, Gclc and Gclm.

Acknowledgments This work was supported by a KAKENHI Grant-in-Aid for Scientific Research (A) (No. 16H02667 IK) and partially supported by a KAKENHI Grantin-Aid for Young Scientists (No. 18K18190 MY).

Conflict of Interest The authors declare no conflict of interest.

\section{REFERENCES}

1) Pollycove M, Feinendegen LE. Radiation-induced versus endogenous DNA damage: possible effect of inducible protective responses in mitigating endogenous damage. Hum. Exp. Toxicol., 22, 290-306, discussion, 307, 315-317, 319-323 (2003).

2) Mikkelsen RB, Wardman P. Biological chemistry of reactive oxygen and nitrogen and radiation-induced signal transduction mechanisms. Oncogene, 22, 5734-5754 (2003).

3) Ishii T, Itoh K, Takahashi S, Sato H, Yanagawa T, Katoh Y, Bannai $\mathrm{S}$, Yamamoto $\mathrm{M}$. Transcription factor Nrf2 coordinately regulates a group of oxidative stress-inducible genes in macrophages. J. Biol. Chem., 275, 16023-16029 (2000).

4) Kensler TW, Wakabayashi N, Biswal S. Cell survival responses to environmental stresses via the keap1-Nrf2-ARE pathway. Annu. Rev. Pharmacol. Toxicol., 47, 89-116 (2007).

5) Kaspar JW, Niture SK, Jaiswal AK. Nrf2:INrf2 (Keap1) signaling in oxidative stress. Free Radic. Biol. Med., 47, 1304-1309 (2009).

6) McDonald JT, Kim K, Norris AJ, Vlashi E, Phillips TM, Lagadec C, Della Donna L, Ratikan J, Szelag H, Hlatky L, McBride WH. Ionizing radiation activates the Nrf2 antioxidant response. Cancer Res., 70, 8886-8895 (2010).

7) Vomund S, Schäfer A, Parnham MJ, Brüne B, von Knethen A. Nrf2, the master regulator of anti-oxidative responses. Int. J. Mol. Sci., 18, 2772 (2017).

8) Lu MC, Ji JA, Jiang ZY, You QD. The Keap1-Nrf2-ARE pathway as a potential preventive and therapeutic target: an update. Med. Res. Rev., 36, 924-963 (2016)

9) Miura S, Yamaguchi M, Yoshino H, Nakai Y, Kashiwakura I. Dosedependent increase of Nrf2 target gene expression in mice exposed to ionizing radiation. Radiat. Res., 191, 176-188 (2019).

10) Yamaguchi M, Hirouchi T, Yoshioka H, Watanabe J, Kashiwakura I. Diverse functions of the thrombopoietin receptor agonist romiplostim rescue individuals exposed to lethal radiation. Free Radic. Biol. Med., 136, 60-75 (2019).

11) Bunin DI, Bakke J, Green CE, Javitz HS, Fielden M, Chang PY. Romiplostim (Nplate $\left.{ }^{B}\right)$ ) as an effective radiation countermeasure to improve survival and platelet recovery in mice. Int. J. Radiat. Biol., 96, 145-154 (2020).

12) Wong K, Chang PY, Fielden M, Downey AM, Bunin D, Bakke J, Gahagen J, Iyer L, Doshi S, Wierzbicki W, Authier S. Pharmacodynamics of romiplostim alone and in combination with pegfilgrastim on acute radiation-induced thrombocytopenia and neutropenia in non-human primates. Int. J. Radiat. Biol., 96, 155-166 (2020).

13) Lefrançais E, Ortiz-Muñoz G, Caudrillier A, Mallavia B, Liu F, Sayah DM, Thornton EE, Headley MB, David T, Coughlin SR, Krummel MF, Leavitt AD, Passegue E, Looney MR. The lung is a site of platelet biogenesis and a reservoir for haematopoietic progenitors. Nature, 544, 105-109 (2017)

14) Nguyen T, Nioi P, Pickett CB. The Nrf2-antioxidant response element signaling pathway and its activation by oxidative stress.
J. Biol. Chem., 284, 13291-13295 (2009).

15) Hirouchi $T$, Ito $K$, Nakano $M$, Monzen $S$, Yoshino $H$, Chiba $M$, Hazawa M, Nakano A, Ishikawa J, Yamaguchi M, Tanaka K, Kashiwakura I. Mitigative effects of a combination of multiple pharmaceutical drugs on the survival of mice exposed to lethal ionizing radiation. Curr. Pharm. Biotechnol., 17, 190-199 (2015).

16) Yamaguchi M, Hirouchi T, Yokoyama K, Nishiyama A, Murakami S, Kashiwakura I. The thrombopoietin mimetic romiplostim leads to the complete rescue of mice exposed to lethal ionizing radiation. Sci. Rep., 8, 10659 (2018).

17) Siegel D, Gustafson DL, Dehn DL, Han JY, Boonchoong P, Berliner LJ, Ross D. NAD(P)H: quinone oxidoreductase 1: role as a superoxide scavenger. Mol. Pharmacol., 65, 1238-1247 (2004).

18) Jaiswal AK. Regulation of genes encoding NAD(P)H:quinone oxidoreductases. Free Radic. Biol. Med., 29, 254-262 (2000).

19) Pey AL, Megarity CF, Timson DJ. NAD(P)H quinone oxidoreductase (NQO1): an enzyme which needs just enough mobility, in just the right places. Biosci. Rep., 39, BSR20180459 (2019).

20) Asher G, Lotem J, Cohen B, Sachs L, Shaul Y. Regulation of p53 stability and p53-dependent apoptosis by NADH quinone oxidoreductase 1. Proc. Natl. Acad. Sci. U.S.A., 98, 1188-1193 (2001).

21) Ross D, Siegel D. NQO1 in protection against oxidative stress. Curr. Opin. Toxicol., 7, 67-72 (2018).

22) Iles KE, Liu RM. Mechanisms of glutamate cysteine ligase (GCL) induction by 4-hydroxynonenal. Free Radic. Biol. Med., 38, 547$556(2005)$.

23) Solis WA, Dalton TP, Dieter MZ, Freshwater S, Harrer JM, He L, Shertzer HG, Nebert DW. Glutamate-cysteine ligase modifier subunit: mouse Gclm gene structure and regulation by agents that cause oxidative stress. Biochem. Pharmacol., 63, 1739-1754 (2002).

24) Mohar I, Botta D, White CC, McConnachie LA, Kavanagh TJ. Glutamate cysteine ligase (GCL) transgenic and gene-targeted mice for controlling glutathione synthesis. Curr Protoc Toxicol., 39, 6.16.1-6.16.20 (2009)

25) Hongmei Z, Haihua R, Hongtao LI. Progress in the research of GSH in cells. Chin. Sci. Bull., 56, 3057-3063 (2011).

26) Krejsa CM, Franklin CC, White CC, Ledbetter JA, Schieven GL, Kavanagh TJ. Rapid activation of glutamate cysteine ligase following oxidative stress. J. Biol. Chem., 285, 16116-16124 (2010).

27) Sekhar KR, Freeman ML. Nrf2 promotes survival following exposure to ionizing radiation. Free Radic. Biol. Med., 88 (Pt. B), 268-274 (2015)

28) Chai J, Luo L, Hou F, Fan X, Yu J, Ma W, Tang W, Yang X, Zhu J, Kang W, Yan J, Liang H. Agmatine reduces lipopolysaccharidemediated oxidant response via activating PI3K/Akt pathway and upregulating Nrf2 and HO-1 expression in macrophages. PLOS ONE, 11, e0163634 (2016).

29) Thabet NM, Moustafa EM. Protective effect of rutin against brain injury induced by acrylamide or gamma radiation: role of PI3K/AKT/GSK-3 $\beta /$ NRF-2 signalling pathway. Arch. Physiol. Biochem., 124, 185-193 (2018).

30) Sandberg M, Patil J, D’Angelo B, Weber SG, Mallard C. NRF2regulation in brain health and disease: implication of cerebral inflammation. Neuropharmacology, 79, 298-306 (2014).

31) Drachman JG, Millett KM, Kaushansky K. Thrombopoietin signal transduction requires functional JAK2, not TYK2. J. Biol. Chem., 274, 13480-13484 (1999).

32) Scapagnini G, Vasto $S$, A braham NG, Caruso C, Zella D, Fabio G. Modulation of Nrf2/ARE pathway by food polyphenols: a nutritional neuroprotective strategy for cognitive and neurodegenerative disorders. Mol. Neurobiol., 44, 192-201 (2011).

33) Zheng Y, Tao S, Lian F, Chau BT, Chen J, Sun G, Fang D, Lantz $\mathrm{RC}$, Zhang DD. Sulforaphane prevents pulmonary damage in response to inhaled arsenic by activating the Nrf2-defense response. Toxicol. Appl. Pharmacol., 265, 292-299 (2012).

34) Satoh T, McKercher SR, Lipton SA. Nrf2/ARE-mediated antioxi- 
dant actions of pro-electrophilic drugs. Free Radic. Biol. Med., 65 , 645-657 (2013)

35) Abdel-Magied N, Shedid SM. The effect of naringenin on the role of nuclear factor (erythroid-derived 2)-like2 (Nrf2) and haem oxygenase 1 (HO-1) in reducing the risk of oxidative stress-related radiotoxicity in the spleen of rats. Environ. Toxicol., 34, 788-795 (2019).
36) Lu L, Dong J, Li D, Zhang J, Fan S. 3,3-Diindolylmethane mitigates total body irradiation-induced hematopoietic injury in mice. Free Radic. Biol. Med., 99, 463-471 (2016).

37) Li Y, Ma S, Zhang Y, Yao M, Zhu X, Guan F. (-)-Epicatechin mitigates radiation-induced intestinal injury and promotes intestinal regeneration via suppressing oxidative stress. Free Radic. Res., 53, 851-864 (2019). 